\title{
Determinación experimental de la velocidad de caída de sedimentos no cohesivos
}

\author{
Experimental determination of fall velocity of \\ non-cohesive sediment \\ Bruno César Comini de Andrade ${ }^{1} \quad$ Jorge Luis Zegarra Tarqui ${ }^{2 *}$ \\ Recibido 29 de junio de 2017, aceptado 31 de enero de 2019 \\ Received: June 29, 2017 Accepted: January 31, 2019 \\ RESUMEN
}

\begin{abstract}
La determinación de la velocidad de caída de partículas no cohesivas (arena) fue medida experimentalmente mediante el empleo de una técnica de filmación con una cámara fotográfica comercial. El diámetro de los sedimentos varió entre $0,075 \mathrm{~mm}$ a $2 \mathrm{~mm}$, los valores experimentales fueron comparados con los valores calculados por ecuaciones empíricas, cuatro ecuaciones consideran solo el diámetro para el cálculo de la velocidad de caída, la quinta ecuación considera el diámetro y el factor de forma (cfs), y la sexta ecuación considera diámetro, factor de forma y la redondez (P). Los resultados mostraron que la técnica de filmación permite medir de forma adecuada la velocidad de caída y que en una faja de diámetro de sedimento entre $0,075 \mathrm{~mm}$ a $1,2 \mathrm{~mm}$, la ecuación que incluye los factores de forma y redondez tuvo uno de los mejores desempeños.
\end{abstract}

Palabras clave: Sedimento, factor de forma, redondez, partícula no cohesiva, velocidad de caída.

\begin{abstract}
The determination of the fall velocity of non-cohesive particles (sand) was measured experimentally by using a technique of filming with a commercial camera. The diameters of the sediment ranged from $0.075 \mathrm{~mm}$ to $2 \mathrm{~mm}$, the experimental values were compared with the ones calculated by empirical equations, four equations only consider the diameter to calculate the falling speed, the fifth equation considers diameter and form factor ( $c f s)$, and sixth equation considers diameter, form factor, and roundness $(P)$. The results showed that the filming technique allows measuring the falling speed adequately and a sediment diameter range from $0.075 \mathrm{~mm}$ to $1.2 \mathrm{~mm}$, the equation that includes the form factors and roundness had one of the best performances.
\end{abstract}

Keywords: Sediment, form factor, roundness, non-cohesive particle, fall velocity.

1 Ingeniería Ambiental. Universidade Federal de Minas Gerais. Av. Antônio Carlos 6627 - Campus da UFMG - Pampulha Belo Horizonte - MG - CEP: 31270-901. E-mail: bruno-cca@ hotmail.com

2 Departamento de Engenharia Hidráulica y Recursos Hídricos. Centro de Pesquisas Hidráulicas e Recursos Hídricos - CPH. Sala G 406. Universidade Federal de Minas Gerais. Av. Antônio Carlos 6627 - Campus da UFMG - Pampulha Belo Horizonte - MG - CEP: 31270-901. E-mail: jlztarqui@yahoo.com.br

* Autor de correspondencia: jlztarqui@yahoo.com.br 


\section{INTRODUCCIÓN}

La determinación de la velocidad de caída de partículas es esencial para estudios de transporte de masa, tratamiento de aguas, preparación de mezclas y otras aplicaciones en la Ingeniería.

Una partícula que sedimenta a velocidad constante $\left(\omega_{s}\right)$ a través de un fluido sufre la acción de tres fuerzas: Arrastre $\left(F_{D}\right)$, Flotación $\left(F_{b}\right)$ y Peso $\left(F_{W}\right)$, como muestra la Figura 1.

Así,

$$
F_{W}=F_{b}+F_{D}
$$

y las fuerzas son definidas como:

$$
\begin{gathered}
F_{W}=\frac{\pi}{6} d_{s}^{3} \rho s g \\
F_{b}=\frac{\pi}{6} d_{s}^{3} \rho f g \\
F_{D}=\pi \mu d_{s} \omega_{s}
\end{gathered}
$$

Donde, $d_{s}$ es el diámetro; $\rho_{s}$ es la densidad del sedimento; $\rho_{f}$ es la densidad del fluido; $\mu$ es la viscosidad del fluido; $g$ es la gravedad. La ecuación (4) representa la fuerza de arrastre para un fluido viscoso en régimen laminar.

Por otro lado, la fuerza de arrastre también es definida de forma general como,

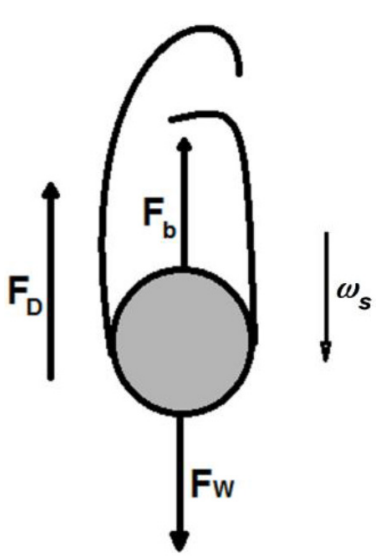

Figura 1. Fuerzas que actúan sobre partícula que sedimenta a velocidad constante $\left(\omega_{s}\right)$.

$$
F_{D}=C_{D} \frac{\rho f \pi \mu_{\infty}^{2} d_{s}^{2}}{8}
$$

Donde $C_{D}$ es el coeficiente de arrastre; y $u_{\infty}$ es la velocidad del fluido pero en caso de fluido en reposo se equipara a la velocidad de caída $\left(\omega_{\mathrm{s}}\right)$.

$\mathrm{Al}$ igualar las ecuaciones (4) y (5) es obtenido el valor de $C_{D}$,

$$
C_{D}=\frac{24}{\operatorname{Re}_{p}}
$$

Donde $\operatorname{Re}_{p}=\frac{\rho_{s} \omega_{s} d_{s}}{\mu}$ es denominado número de Reynolds de la partícula. Por otro lado, substituyendo las ecuaciones (2), (3) y (4) en la ecuación (1), se obtiene la velocidad de caída [1].

$$
\omega_{s}=\frac{g}{18} \frac{\rho_{s}-\rho_{f}}{\mu} d_{s}^{2}
$$

Entretanto, resultados experimentales mostraron que la ecuación (7) solo se aplica a partículas esféricas y número de Reynolds de partícula inferiores a 1. Con valores de $R e_{p}$ superiores a 1 aparecen vórtices afectando el valor del coeficiente de arrastre. Otras fórmulas empíricas fueron creadas en la tentativa de abarcar una faja mayor de Reynolds, considerando partículas naturales, en ese sentido [2] propone una ecuación general de $C_{D}$.

$$
C_{D}=\left[\left(\frac{A}{\operatorname{Re}_{p}}\right)^{1 / m}+B^{1 / m}\right]^{m}
$$

Donde, $A$ y $B$ son coeficientes y $m$ es un exponente, así varios autores proponen valores de $A, B$ y $m$. Ver Tabla 1.

Las ecuaciones de [3-4-5], junto a otras como [7-89], tienen una base empírica y son adecuadas para $1<R e_{p}<5000$. Estas ecuaciones están en función de diámetro de la partícula y no consideran los efectos de la forma sobre la velocidad de caída [6]. El coeficiente de forma (csf) es la razón entre area superficial de una esfera y area superficial 
Tabla 1. Valores de $A, B$ y $m$.

\begin{tabular}{|l|l|l|l|}
\hline \multicolumn{1}{|c|}{ Autor } & \multicolumn{1}{c|}{ A } & \multicolumn{1}{c|}{ B } & \multicolumn{1}{c|}{ m } \\
\hline Cheng [3] & 32 & 1 & 1,5 \\
\hline Rubey [4] & 24 & 2 & 1 \\
\hline Julien [5] & 24 & 1,5 & 1 \\
\hline Camenen [6] & 24,6 & 0,96 & 1,53 \\
\hline
\end{tabular}

Nota: Adaptación de [3].

de un grano del mismo volumen. Cuando el valor de $c s f$ se aproxima a la unidad, más esférica es la partícula; cuanto menor el csf, más alargada es la partícula. La redondez $(P)$ mide la cantidad de aristas de una partícula y cuanto de puntiagudas son. Existen varios métodos para la determinación de las características mencionadas, pero la obtención de valores precisos es compleja y sujeta a grandes variaciones.

La ecuación propuesta por [10] considera un polinomio de cuarto grado, lo que la vuelve compleja para su uso, posteriormente [11] proponen otra ecuación con diferentes coeficientes para especificar el factor de forma y la redondez. Luego [6] propone una ecuación donde los coeficientes $A, B$ y m están en función del factor $c f s$ y $P$.

El presente estudio tuvo por objetivo encontrar un método barato y simple para determinar la velocidad de caída de partículas de arena, con diámetro entre $0.075 \mathrm{~mm}$ y $2.00 \mathrm{~mm}$. Así como evaluar los valores experimentales con los valores calculados por las ecuaciones propuestas por $[1,3-4,6]$.

\section{METODOLOGIA}

\section{Determinación de Granulometría y Masa Específica}

La arena fue del rio Paraopeba, Estado de Minas Gerais, Brasil. El material fue lavado y después tamizado utilizando mallas con los siguientes diámetros de abertura: \# $2.00 \mathrm{~mm}$; \# $1.20 \mathrm{~mm}$; \# $0.6 \mathrm{~mm}$; \# $0.42 \mathrm{~mm}$; \# $0.25 \mathrm{~mm}$; \# $0.15 \mathrm{~mm}$ y \# $0.075 \mathrm{~mm}$. En la Tabla 2 se encuentra la masa retenida en cada tamiz del ensayo de distribución granulométrica. El diámetro medio de cada intervalo fue calculado a partir de la media aritmética de los diámetros que delimitan cada intervalo. La densidad de la arena fue de $2,874.73 \mathrm{~kg} / \mathrm{m}^{3}$, determinada en laboratorio siguiendo las Normas Técnicas Brasileñas.

\section{Montaje del Ensayo}

Se Construyó un decantador en acrílico, de $2 \mathrm{~m}$ de altura con sección transversal cuadrada de $0,4 \mathrm{~m} \times 0,4 \mathrm{~m}$, instalado sobre una estructura metálica como base. El equipamiento fue llenado completamente con agua y colocado entre una cámara fotográfica y un fondo blanco (Figura 2). Los experimentos fueron realizados en los mismos horarios del día, con temperatura media de $20^{\circ} \mathrm{C}$, con la finalidad de evitar variación del coeficiente de viscosidad del fluido y afectar la velocidad de caída, como fue observado por [12].

Tabla 2. Masa retenida por tamiz en el ensayo de distribución granulométrica.

\begin{tabular}{|c|c|c|}
\hline $\begin{array}{c}\text { Intervalo } \\
(\mathbf{m m})\end{array}$ & $\begin{array}{c}\text { Diámetro } \\
\text { medio } \\
(\mathbf{m m})\end{array}$ & $\begin{array}{c}\text { Masa } \\
\text { Retenida } \\
(\mathbf{g r})\end{array}$ \\
\hline$<2.00-1.20>$ & 1.6 & 14.52 \\
\hline$<1.20-0.60>$ & 0.9 & 224.18 \\
\hline$<0.60-0.42>$ & 0.51 & 295.00 \\
\hline$<0.42-0.25>$ & 0.33 & 239.30 \\
\hline$<0.25-0.15>$ & 0.2 & 113.60 \\
\hline$<0.15-0.075>$ & 0.1125 & 84.98 \\
\hline Fundo & - & 27.88 \\
\hline
\end{tabular}

Para la filmación se usaron dos cámaras fotográficas semi profesionales con la finalidad de mejorar la resolución de las imágenes de video, conforme la faja granulométrica experimentada. Cada cámara fue posicionada de forma paralela al decantador, se utilizó una regla escuadra para colocar el lente de la cámara paralelo a la pared de acrílico del decantador, la distancia media entre el lente y el eje central del decantador fue de $25 \mathrm{~cm}$, aproximadamente. El área de filmación se encuentra, aproximadamente, en la parte inferior del decantador, como es presentado en la Figura 2.

En la parte inferior del fondo blanco se instaló una lámpara de iluminación, con la finalidad de generar un máximo contraste en la imagen capturada por la cámara [13-14]. La parte inferior del decantador tiene un formato de tronco de pirámide, lo que facilita el drenaje del mismo una vez finalizado el experimento. 


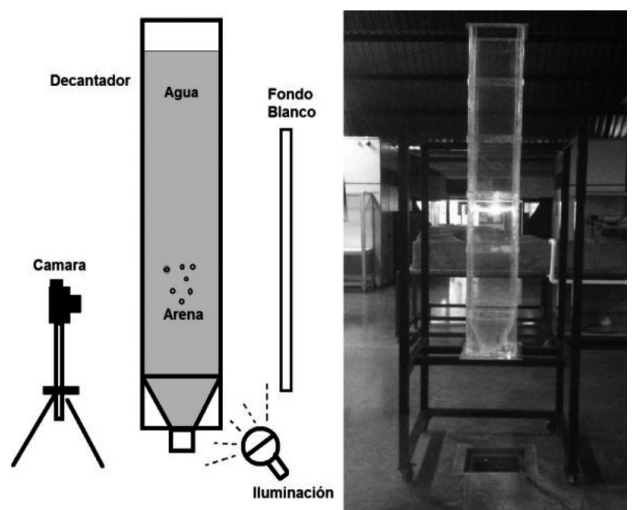

Figura 2. Diseño esquemático del decantador (a la izquierda); Foto del decantador (a la derecha).

\section{Adquisición y procesamiento de datos}

Una cantidad entre 3 y 5 gramos de arena fue colocada de forma lenta en la parte superior del decantador y su trayecto fue grabado, se observó una columna de desplazamiento de partículas larga y dispersa, de esta forma se disminuye el efecto de la concentración. Después de la filmación, los vídeos fueron editados con la finalidad de maximizar el contraste entre las partículas y el fondo blanco.

Luego, las imágenes fueron seleccionadas de forma secuencial, cuadro por cuadro, pero con un intervalo de tiempo predefinido entre ellas (30 o 60 cuadros por segundo). Los cuadros secuenciales fueron grabados y tratados por un software libre, que permitió medir el recorrido de la partícula para cada intervalo de tiempo, como muestra la Figura 3.

Dentro del decantador fue colocada una barra metálica que ayudó en la focalización de las imágenes y sirvió para la calibración de la escala de medición facilitando
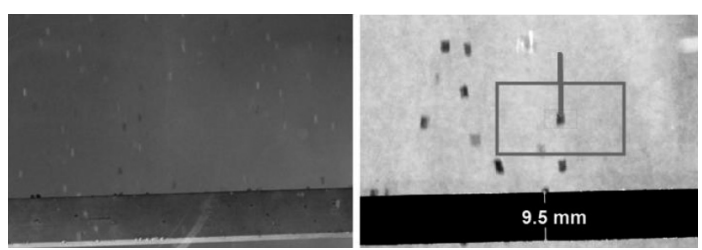

Figura 3. Imagen original (izquierda) e imagen con contraste (derecha) con trazado secuencial del recorrido de una partícula. En ambas imágenes, detalle de la barra metálica para la calibración. la determinación de las distancias recorridas por las partículas de arena filmadas. Con base en la distancia recorrida y el intervalo de tiempo existente entre cuadro y cuadro fueron calculadas diferentes velocidades instantáneas de caída para una única partícula, permitiendo después calcular la velocidad media de la partícula analizada, este mismo procedimiento fue aplicado para otras partículas dentro de varios videos de filmación para un mismo intervalo granulométrico, obteniendo un conjunto de velocidades medias para diferentes partículas.

Para cada conjunto de velocidades medias de diferentes partículas, referente a un intervalo granulométrico de arena, se calculó la velocidad media, la mediana y su desviación estándar. Los resultados de velocidad mediana fueron comparados con valores de velocidad determinadas por las ecuaciones propuestas más adelante. Con la finalidad de una mejor identificación serán empleados los nombres de los autores para designar las ecuaciones.

\section{Ecuaciones empleadas}

La ecuación de Stokes [1], como fue mencionado anteriormente, aplicada a partículas esfericas y $R e_{p}<1$ :

$$
\omega_{s}=\frac{1}{18} \frac{\left(\rho_{s}-\rho_{f}\right)}{\mu} g d_{s}^{2}
$$

$\omega_{s}$ es la velocidad de sedimentación; $\mu$ es la viscosidad dinámica del fluido; $\rho_{f}$ es la densidad del fluido; $\rho_{s}$ es la densidad de la partícula de sedimento; $d_{s}$ es el diámetro; $G$ es la densidad relativa de la partícula, $g$ es la aceleración gravitacional.

La ecuación de Rubey [4] fue obtenida con una aproximación sencilla del coeficiente $C_{D}$,

$$
D_{D}=\frac{24}{\operatorname{Re}_{p}}+2
$$

$\mathrm{Y}$ es definida como,

$$
\omega_{s}=\left[\left(\frac{2}{3}\right)+\frac{36}{d_{s}^{3}}-\frac{36}{d_{*}^{3}}\right] \sqrt{(G-1) g d_{s}}
$$

Donde a $d_{*}$ es definido como el diámetro adimensional de partícula. 


$$
d_{*}=d_{s}\left[\frac{(G-1) g}{\vartheta^{2}}\right]^{1 / 3}
$$

Esta ecuación fue desarrollada para agua limpia y su uso es raro, excepto en la ecuación de transporte por arrastre de Einstein conforme menciona [5].

La ecuación de Cheng [3] es definida como,

$$
\omega_{S}=\frac{\vartheta}{d_{S}}\left[\left(25+1.2 d_{s}^{2}\right)^{0.5}-5\right]^{1.5}
$$

Por otro lado Julien sugiere una oproximación del coeficiente $C_{D}$

$$
C_{D}=\breve{C}_{D}+\frac{24}{\operatorname{Re}_{p}}
$$

Entonces la ecuación original de Julien [5] es definida como,

$$
\omega_{s}=12 \frac{\vartheta}{d_{s} \breve{C}_{D}}\left[\left(1+\frac{\breve{C}_{D}}{108} d_{*}^{3}\right)^{1 / 2}-1\right]
$$

Donde $\check{C}_{D}=15$ para gravas y arenas naturales. $\mathrm{Si}$ sustituimos este valor en la ecuación original se llega a la ecuación de Julien utilizada en este estudio.

$$
\omega_{s}=8 \frac{\vartheta}{d_{s}}\left[\left(1+0.0139 d_{*}^{3}\right)^{1 / 2}-1\right]
$$

La ecuación de Camenen [6] es definida como,

$$
\omega_{s}=\frac{\mu}{\rho_{f} d_{s}} \operatorname{Re}_{p}
$$

Donde,

$$
\operatorname{Re}_{p}=\left[\sqrt{\frac{1}{4}\left(\frac{A}{B}\right)^{2 / m}+\left(\frac{4 d_{*}^{3}}{3 B}\right)^{1 / m}}-\frac{1}{2}\left(\frac{A}{B}\right)^{1 / m}\right]^{m}
$$

las variables son definidas como,

$$
\begin{gathered}
A=a_{1}+a_{2}\left[1-\operatorname{sen}\left(\frac{\pi}{2} c f s\right)\right]^{a_{2}} \\
B=b_{1}+b_{2}\left[1-\operatorname{sen}\left(\frac{\pi}{2} c f s\right)\right]^{b_{2}} \\
m=(1.2+0.12 P) \operatorname{Sen}^{0.47}\left(\frac{\pi}{2} c f s\right)
\end{gathered}
$$

$a_{1}=24 ; a_{2}=100 ; a_{3}=2.1+0.06 P$

$b_{1}=0.39+0.22(6+P) ; b_{2}=20$

$b_{2}=0.75+0.3 P$

Los valores adoptados de $\operatorname{csf}$ y $P$ para arenas varían entre $<0.5$ y $0.9>$ y $<2.0$ y $3.5>$, respectivamente, [6] sugiere los valores $c s f=0.7$ y $P=3.5$, que corresponde a arenas de lecho de río.

Un estudio comparativo de evaluación de velocidad de caída de sedimentos naturales [15] se observó que la ecuación de Wu y Wang [16] y Cheng [3] reproducen mejor los datos experimentales.

La ecuación de Wu y Wang [16] es definida como,

$$
\omega_{s}=\frac{M}{N} \frac{\vartheta}{d_{s}}\left[\sqrt{\frac{1}{4}+\left(\frac{4 N}{3 M^{2}} d_{*}^{3}\right)^{1 / n}}-\frac{1}{2}\right]^{n}
$$

Donde

$N=5.65 e^{-25 c f s} M=53.6 e^{-0.65 c f s}$

$n=0.7+0.9 c f s$

Se observa, que a diferencia de la ecuación de Camenen, la ecuación de Wu y Wang solo considera el factor de forma y no la redondez.

Los valores $c f s$ y $P$ fueron obtenidos experimentalmente usando el método de determinación bidimensional, [17] para el factor de forma y [18] para a redondez.

La estimación bidimensional de los parámetros es calculada por las ecuaciones (15) y (16), a seguir:

$$
\begin{gathered}
c f s=\sqrt{\frac{D_{i}}{D_{c}}} \\
P=6 x \frac{\sum_{i=1}^{n} r_{i}}{R n}
\end{gathered}
$$


$D_{i}$ es el diámetro de la mayor circunferencia inscrita al sedimento; $\mathrm{R}$ es el radio de la misma circunferencia; $D_{c}$ es el diámetro de la circunferencia circunscrita al sedimento; $r_{i}$ es el radio de la circunferencia correspondiente a cada vértice; $n$ es el número de vértices del sedimento. La Figura 4 presenta una representación esquemática de la disposición de las circunferencias sobre la imagen del sedimento.

La Figura 5 presenta los granos de arena dispersos sobre el plano blanco, se percibe una variación en la forma, coloración y tonalidad de los granos de arena, lo que evidencia su diferente composición mineralógica. Para edición de las imágenes, fue utilizado el software libre, AutoCAD (versión estudiante).

Como criterio de comparación de los resultados experimentales $\left(V_{\text {exp }}\right)$ con las valores calculados $\left(V_{c a l}\right)$ por las ecuaciones mencionadas, será utilizada la regresión linear,
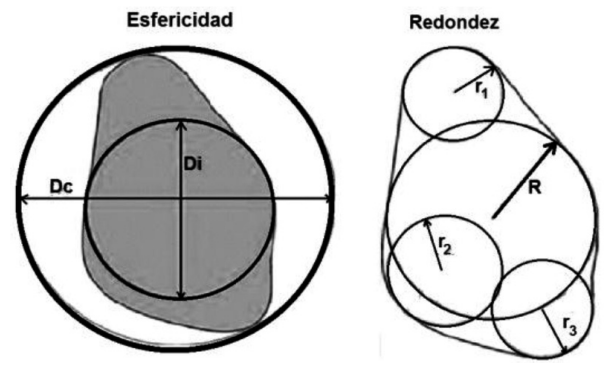

Figura 4. Diseño esquemático de la disposición de las circunferencias sobre el sedimento, necesarias para el cálculo del factor de forma (esfericidad), a la izquierda, y de la redondez, a la derecha.

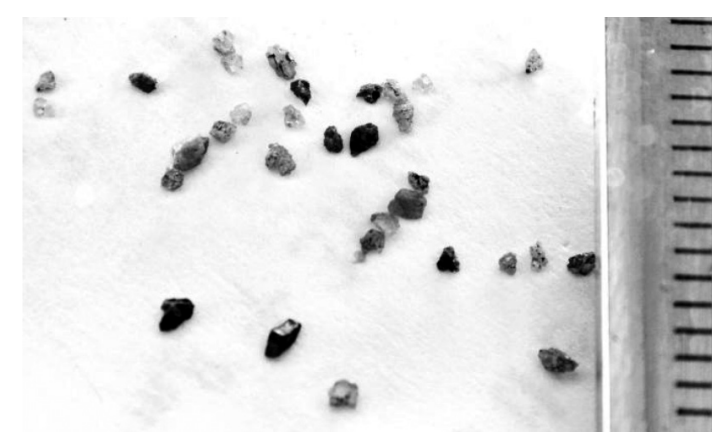

Figura 5. Distribución de los granos de arena para medición de esfericidad y redondez.

$$
V_{\text {exp }}=a V_{c a l}+b
$$

Para un ajuste perfecto esperase que los valores de la inclinación $a$ y de la interceptación $b$ se aproximen a 1 y 0 , respectivamente, entonces se predefine $b=0$ y solo se calcula el valor $a$ en los ajustes de recta mostrados en la Tabla 4.

También, se calcula la Raíz del Error Cuadrático Medio o Root Mean Squared Error (RMSE) que es una medida cuantitativa de desempeño para evaluar los errores entre los valores experimentales y los valores calculados. RMSE amplifica y da mayor peso a aquellos errores de mayor magnitud. La fórmula de cálculo del RMSE es definida como:

$$
R M E S=\sqrt{\frac{\sum_{i=1}^{n}\left(V_{i \exp }-V_{i c a l}\right)^{2}}{n}}
$$

Donde, $n$ es el número de datos, $V_{i \text { exp }}$ es la velocidad experimental, y $V_{\text {ical }}$ es la velocidad calculada por la ecuación utilizada.

Para evaluar la exactitud de las ecuaciones para predecir los valores experimentales fue utilizado el Coeficiente de Eficiencia de Nash-Sutcliff (E), definido como:

$$
E=1-\frac{\sum_{i=1}^{n}\left(V_{i \exp }-V_{i c a l}\right)^{2}}{\sum_{i=1}^{n}\left(V_{i \exp }=\bar{V}_{\exp }\right)^{2}}
$$

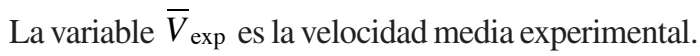
Un valor $E$ más próximo a la unidad representa una mejor capacidad de predicción de la ecuación entre los valores experimentales y calculados.

\section{RESULTADOS Y DISCUSIÓN}

Una manguera fue usada para llenar el interior del decantador con agua tratada a partir del fondo, de manera a generar el mínimo de turbulencia en la columna líquida. La temperatura del agua fue monitoreada a lo largo del experimento por medio de un termómetro de mercurio instalado en la superficie líquida.

La cantidad de sedimento fue colocado lentamente en la parte superior del decantador de manera a evitar 
perturbaciones en la entrada y mantener la velocidad de entrada cerca de cero. Las partículas de arena descendieron de forma homogénea, formando una especie de cortina de partículas. La barra metálica colocada en el centro del decantador sirvió para facilitar la focalización, pues las cámaras utilizadas tienen focalización automática.

Se usaron dos cámaras fotográficas semi profesionales, Tabla 3, con diferente resolución y frecuencia de adquisición, segundo el tipo de fenómeno filmado.

Durante la filmación de los experimentos con la cámara (1), se notó la ineficiencia de la misma para capturar imágenes de partículas con diámetro menor que $0.15 \mathrm{~mm}$, con frecuencias de 60 y 30 cuadro/ segundo. Entonces, se utilizó la cámara (2), con una lente macro hasta 1:1, con la que fue posible capturar en vídeo el movimiento de las partículas con $d_{s}$ menores que $0.15 \mathrm{~mm}$. Con ambos modelos de cámaras se tuvo dificultad con la focalización, pues los modelos realizan este procedimiento de forma automática, lo ideal es usar modelos con focalización manual.

Al principio, fue utilizado el software libre AutoCAD 2012 - Versión Estudiantil, para medir las distancias recorridas por las partículas entre las imágenes secuenciales de los vídeos, este proceso fue considerado laborioso y demorado. Por ese motivo, optase por el software, también libre, Kinovea - Versión 0.8.15, que permite el seguimiento automático y manual de objetos, informa datos como distancia recorrida y velocidad y permite exportar las informaciones para otros programas.

Como criterio de selección de las imágenes, con partículas usadas para medición de la velocidad, se optó por imágenes que presentaron mejor la distribución espacial y mayor distancia de separación entre las partículas. Otro criterio de selección fue desconsiderar las imágenes de partículas que aparecen al principio y al final de toda la filmación.

Tabla 3. Características de las cámaras.

\begin{tabular}{|c|c|c|}
\hline Cámara & $\begin{array}{c}\text { Resolución } \\
\text { (pixeles) }\end{array}$ & $\begin{array}{c}\text { Frecuencia } \\
\text { (cuadro/segundo) }\end{array}$ \\
\hline 1 & $320 \times 240$ & 60 \\
\hline 1 & $640 \times 480$ & 30 \\
\hline 2 & $1920 \times 1080$ & 30 \\
\hline
\end{tabular}

La Tabla 4 presenta un resumen de las etapas de la colecta de datos. También, hubo una reducción del número de vídeos grabados por faja granulométrica, esto se debe a la mejora de la técnica de filmación lo que permitió la visualización de más partículas por vídeo.

Un aspecto limitante del equipamiento es la falta de recurso que permita cambiar la profundidad de campo, lo que impide reducir el número de partículas que aparecen fuera de foco. Una cámara con mayores recursos es más cara, lo que puede inviabilizar su adquisición. A pesar de estas limitaciones, el equipamiento permite obtener imágenes que pueden ser procesadas por el software, sea de forma manual o automática.

La Tabla 5 presenta los seis diferentes intervalos granulométricos definidos y la amplitud de cada intervalo. Para cada intervalo fueron determinados: el número de partículas analizadas, la velocidad media, la velocidad mediana, la desviación estándar, el primer cuartil, el tercer cuartil, el valor mínimo y el valor máximo.

El gráfico Box-plot (Figura 6) permite analizar mejor los resultados, se observa una proximidad entre los valores de la velocidad media y la mediana que permite inferir que las distribuciones están cerca de una distribución casi normal con cierta asimetría.

La variabilidad del primer cuartil y del tercer cuartil, así como la diferencia entre los valores mínimos y máximos puede ser explicada por la composición mineralógica del sedimento, el cual es caracterizado por arena fina y limosa, compuesto por cuarzo y una coloración plomo debido a la contaminación de mineral de hierro y bastante mica. Debido a la dificultad de focalizar los sedimentos de diámetros menores, $d_{s}<0.25 \mathrm{~mm}$, tal vez las imágenes solo capturaron las partículas de sedimento que permiten mejor contraste y mejor reflejo de la iluminación,

Tabla 4. Resumen de las etapas de colecta de datos.

\begin{tabular}{|c|c|c|l|}
\hline Cámara & $\mathbf{d}_{\mathbf{s}}(\mathbf{m m})$ & $\begin{array}{c}\text { Partículas } \\
\text { Analizadas }\end{array}$ & Software \\
\hline 1 & $0.15 \mathrm{a} 2$ & 23 & AutoCAD \\
\hline 2 & $0.075 \mathrm{a} 0.15$ & 3 & AutoCAD \\
\hline 2 & $0.075 \mathrm{a} 2$ & 38 & Kinovea \\
\hline
\end{tabular}


Tabla 5. Caracterización estadística conforme al intervalo granulométrico.

\begin{tabular}{|l|c|c|c|c|c|c|}
\hline Intervalo Granulométrico (mm) & $<0.075-0.15\rangle$ & $\langle 0.15-0.25\rangle$ & $<0.25-0.42\rangle$ & $<0.42-0.6>$ & $<0.6-1.2\rangle$ & $<1.2-2.0\rangle$ \\
\hline $\begin{array}{l}\text { Amplitud del Intervalo } \\
\text { Granulométrico (mm) }\end{array}$ & 0.075 & 0.10 & 0.17 & 0.18 & 0.60 & 0.80 \\
\hline Número de Partículas Analizadas & 8 & 9 & 12 & 12 & 12 & 14 \\
\hline Velocidad mediana (cm/s) & 1.53 & 4.55 & 6.38 & 8.07 & 11.67 & 14.06 \\
\hline Velocidad Media (cm/s) & 1.52 & 4.50 & 6.22 & 8.46 & 11.83 & 14.99 \\
\hline Desviación estándar (cm/s) & 0.38 & 0.32 & 1.58 & 2.11 & 2.10 & 3.14 \\
\hline Primer Cuartil (cm/s) & 1.22 & 4.20 & 4.91 & 7.00 & 10.04 & 13.00 \\
\hline Mínimo (cm/s) & 1.02 & 3.96 & 4.17 & 5.26 & 8.93 & 10.74 \\
\hline Máximo (cm/s) & 2.13 & 5.09 & 8.43 & 12.06 & 15.52 & 20.60 \\
\hline Tercer Cuartil (cm/s) & 1.73 & 4.80 & 7.67 & 9.95 & 12.96 & 16.25 \\
\hline Reynolds de Partícula & 5.71 & 25.17 & 46.68 & 108.85 & 301.10 & 588.61 \\
\hline
\end{tabular}

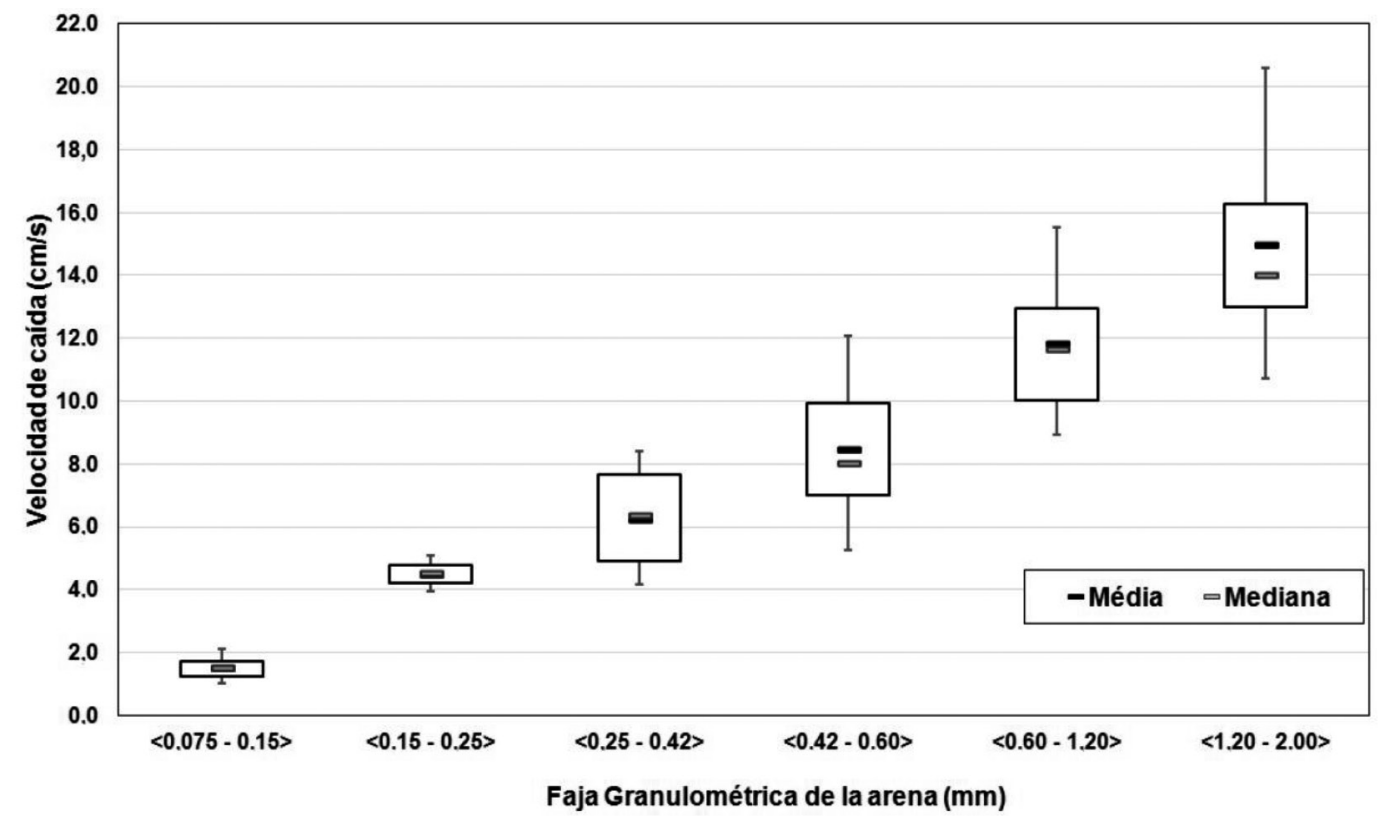

Figura 6. Gráfico Box-plot de la velocidad de caída en función del diámetro medio de arena.

induciendo a filmar partículas con composición mineralógica similar. Con el aumento de la faja de tamaño de las partículas es posible visualizar mayor número de ellas con diferentes coloraciones y tonalidades, por consiguiente mayor variabilidad de composición mineralógica. Otro aspecto que afecta la variabilidad de las mediciones es el aumento de la amplitud del intervalo granulométrico pues con mayor amplitud se tendrá mayor variabilidad de los diámetros presentes en cada intervalo.

Finalmente fue calculado el Número de Reynolds de la partícula de arena con base en el diámetro medio y la velocidad media presente en cada intervalo, se observa que todos los valores son mayores que la unidad, lo que inviabiliza el uso de la ecuación de Stokes.

Recordando que para utilizar la ecuación de Wu y Wang fue necesario determinar los valores de csf, para utilizar la ecuación de Camenen fueron determinados los valores de $\operatorname{csf}$ y $P$. La Tabla 6 presenta los valores de factor de forma y redondez de la arena para cada faja granulométrica.

Las velocidades de caída encontradas en los ensayos experimentales fueron comparadas con los valores 
Tabla 6. Factor de Forma y redondez de los granos de arena por faja granulométrica.

\begin{tabular}{|c|c|c|}
\hline $\begin{array}{c}\text { Faja } \\
\text { Granulométrica }\end{array}$ & Factor de Forma & Redondez \\
\hline$<0.075-0.15>$ & 0.738 & 3.923 \\
\hline$<0.15-0.25>$ & 0.746 & 3.796 \\
\hline$<0.25-0.42>$ & 0.757 & 3.600 \\
\hline$<0.42-0.6>$ & 0.772 & 3.347 \\
\hline$<0.6-1.2>$ & 0.805 & 2.782 \\
\hline$<1.2-2.0>$ & 0.863 & 1.769 \\
\hline
\end{tabular}

de velocidades calculados por las ecuaciones de Stokes, Rubey, Camenen, Julien y Wu y Wang conforme muestran los gráficos de las Figuras 7 y 8. En la Tabla 7 son presentados los valores de $a$, $R M S E$ y $E$, respectivamente.

El coeficiente $\boldsymbol{a}$ de la ecuación de Stokes presenta un valor menor que de la unidad lo que significa que la relación de los valores calculados y experimentales no siguen una relación linear. RMSE y E muestran valores muy altos confirmando la baja capacidad de predicción de la ecuación de Stokes para Reynolds de partícula menores que 1. Comparando los valores de $\boldsymbol{a}, R M S E$ y $E$ obtenidos a partir de las ecuaciones
Tabla 7. Valores de la inclinación (a), Raíz del error cuadrático medio (RMSE) y del Coeficiente de Eficiencia de Nash-Sutcliff $(E)$.

\begin{tabular}{|l|c|c|c|}
\hline \multicolumn{1}{|c|}{ Ecuación } & $\boldsymbol{a}$ & $\boldsymbol{R M S E}(\mathbf{c m} / \mathbf{s})$ & $\boldsymbol{E}$ \\
\hline Stokes & 0.044 & 96.2 & -610.54 \\
\hline Rubey & 1.036 & 1.106 & 0.929 \\
\hline Cheng & 0.825 & 1.526 & 0.846 \\
\hline Camenen & 0.888 & 1.054 & 0.926 \\
\hline Julien & 0.851 & 0.904 & 0.946 \\
\hline Wu y Wang & 0.703 & 0.968 & 0.531 \\
\hline
\end{tabular}

de Rubey, Cheng, Camenen, Julien y Wu y Wang se observa que la ecuación de Julien tiene una mejor predicción de los resultados experimentales, seguido por los resultados de las ecuaciones de Rubey y Camenen. Recordemos que la ecuación de Julien considera un valor de 1.5 específico para gravas y arenas lo que adecúa mejor la ecuación para sedimentos naturales.

El valor de $E$ para la ecuación de Wu y Wang fue debajo de lo esperado, a pesar de ser una ecuación que considera el factor de forma, este resultado puede ser afectado por el método utilizado para el cálculo del factor de forma [17], por otro lado esta ecuación no considera la redondez como la ecuación de Camenen.

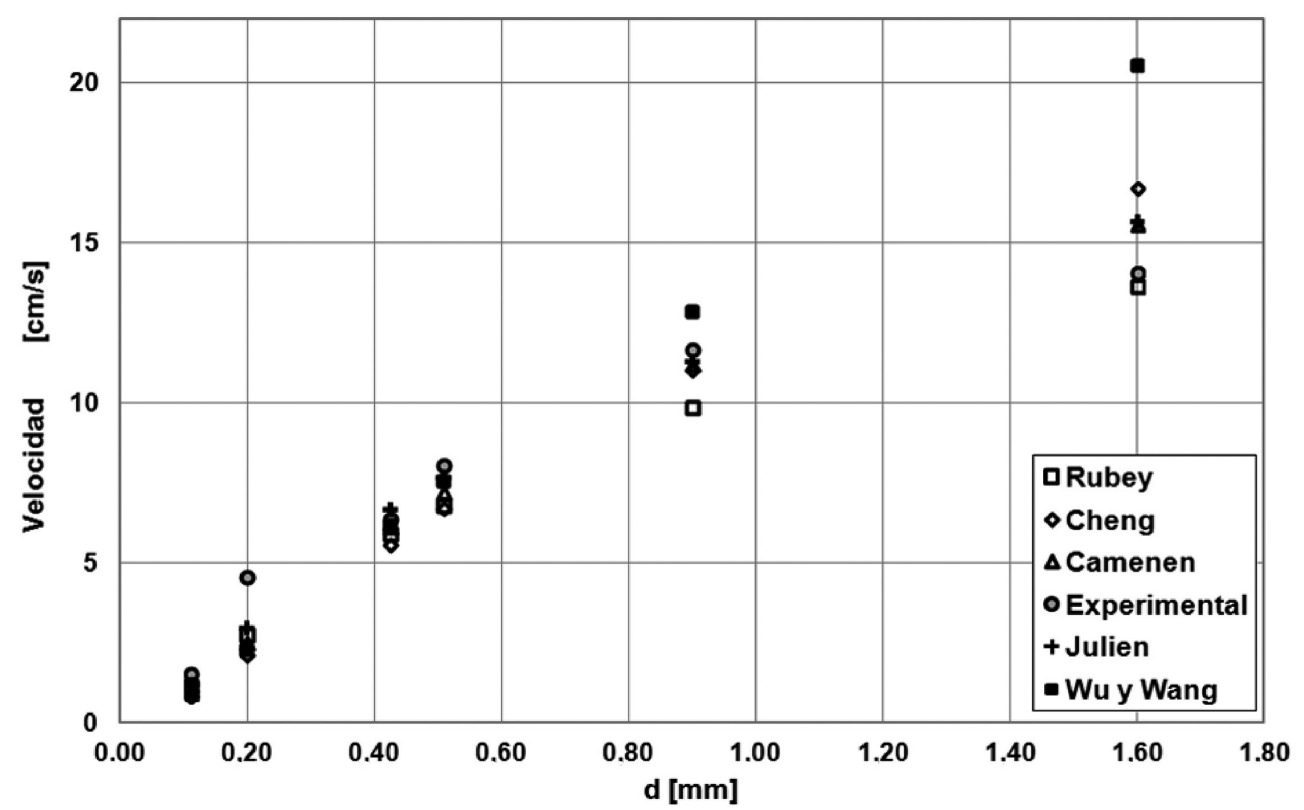

Figura 7. Resultados de las velocidades de caída experimentales y las velocidades calculadas por las ecuaciones de Rubey, Camenen, Cheng Julien y Wu y Wang. 


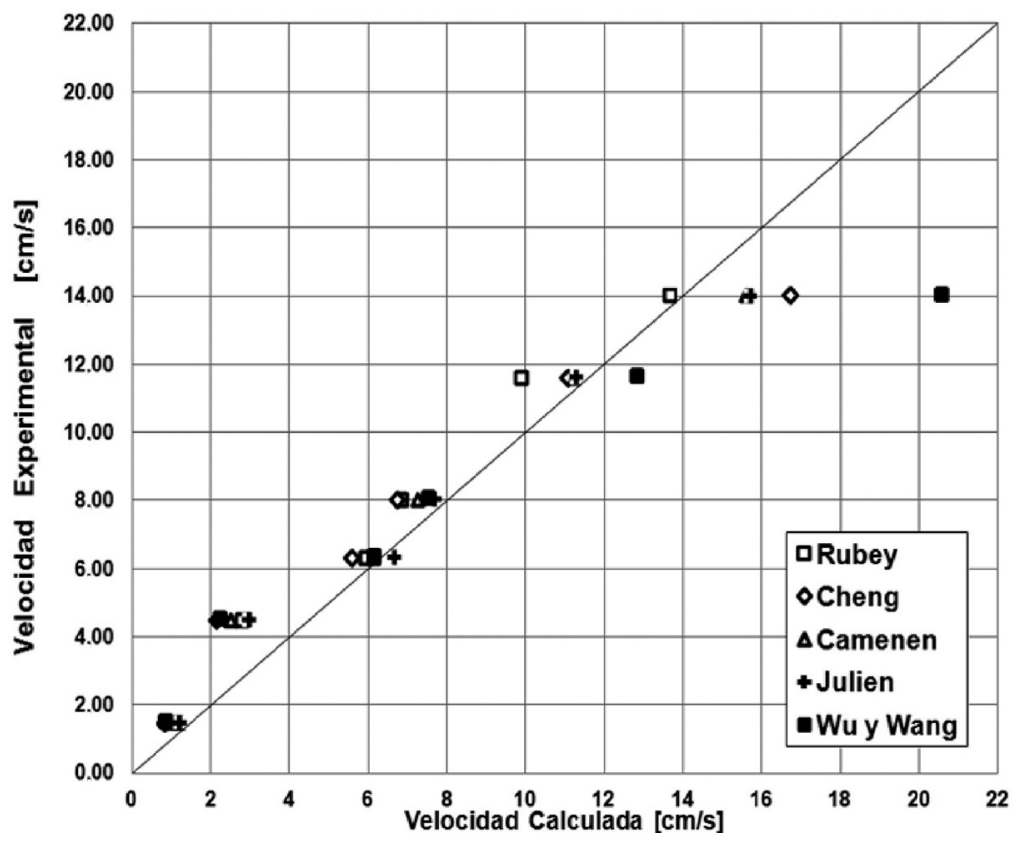

Figura 8. Comparación de la velocidad experimental con la velocidad calculad por las ecuaciones de Rubey, Cheng, Camenen, Julien y Wu y Wang.

En la Figura 7 se visualiza un comportamiento similar de los valores experimentales y los valores calculados por las ecuaciones de Rubey, Cheng, Camenen, Julien y Wu e Wang. También, en la Figura 8, donde son comparados las velocidades experimentales (ordenadas) y velocidades calculadas (abscisas), se constata que la mayoría de los puntos se encuentran levemente arriba de la línea recta con inclinación de $45^{\circ}$ demostrando que la mayoría de los valores calculados subestiman los valores experimentales, una posible explicación es el efecto de la cantidad de partículas sobre las fuerzas de arrastre. Si se descarta la velocidad experimental referente a $d_{s}=1.6 \mathrm{~mm}$, se observa que los valores de velocidad obtenidos por la ecuación de Camenen están muy próximos a la recta de $45^{\circ}$, teniendo un segundo mejor resultado de predicción $(E=0.90)$, pues con la ecuación de Julien se obtuvo un valor de $E=0.94$.

Con la finalidad de evaluar el comportamiento de la velocidad de caída, para $d_{s}=1,6 \mathrm{~mm}$ en otras condiciones, se repitieron los experimentos para temperaturas de fluido de $25^{\circ} \mathrm{C}$ y $27^{\circ} \mathrm{C}$, los resultados son mostrados en la Tabla 8. Un aumento de la temperatura determina una reducción del efecto de la viscosidad y por consiguiente un aumento de la velocidad de caída. Como se observa en la Tabla 7, los valores de velocidad de caída aumentan con el incremento de la temperatura, los valores experimentales de velocidad (de $25^{\circ} \mathrm{C}$ a $27^{\circ} \mathrm{C}$ ) y están más próximos a los valores calculados, entonces se puede afirmar que las ecuaciones de Julien y Camenen son más indicadas para la determinación de la velocidad de caída para el sedimento estudiado.

Tabla 8. Velocidades medias experimentales para $d_{s}=1.6 \mathrm{~mm}$ y velocidades calculadas por la ecuación de Camenen para $20^{\circ} \mathrm{C}, 25^{\circ} \mathrm{C}$ y $27^{\circ} \mathrm{C}$.

\begin{tabular}{|l|c|c|c|}
\hline \multicolumn{1}{|c|}{ Temperatura } & $\mathbf{2 0}^{\mathbf{}} \mathbf{C}$ & $\mathbf{2 5}^{\mathbf{}} \mathbf{C}$ & $\mathbf{2 7}^{\mathbf{}} \mathbf{C}$ \\
\hline Velocidad media Experimental (cm/s) & 14.92 & 16.92 & 16.29 \\
\hline Velocidad calculada (Camenen) & 15.58 & 15.70 & 15.75 \\
\hline Velocidad calculada (Julien) & 15.69 & 15.74 & 15.76 \\
\hline
\end{tabular}

Posteriormente se hará un estudio evaluando el efecto de la temperatura sobre la velocidad de caída.

Se sabe que existen diversos factores que pueden afectar en menor o mayor grado los resultados. Un factor que puede explicar las diferencias entre 
los valores experimentales y calculados es el uso $d_{s}=1.6 \mathrm{~mm}$, valor medio dentro del intervalo granulométrico de $1.2 \mathrm{~mm}$ y $2 \mathrm{~mm}$, con valor de amplitud $(0.8 \mathrm{~mm})$ mayor que de los otros intervalos granulométricos. La falta de tamices entre las mallas de abertura de 1.2 a $2 \mathrm{~mm}$ impidió definir más intervalos granulométricos y reducir la amplitud.

$\mathrm{Al}$ contrario, los valores calculados fueron obtenidos aplicando las ecuaciones (3), (4) y (6) ajustadas con datos experimentales de partículas individuales de arena y en algunos casos usando esferas [4]. Otro factor a considerar es la composición mineral heterogénea del sedimento, que provoca valores de diferentes velocidades para partículas con la misma granulometría.

\section{CONCLUSIONES}

El método de visualización mediante la filmación, usando una cámara fotográfica, es menos sofisticado, barato y permite medir adecuadamente la velocidad. Sin embargo, es relativamente laborioso especialmente en la etapa de procesamiento manual de las imágenes, pero eso depende del tipo de software utilizado, lo que facilita esta etapa. Este método es restricto para medición da velocidad de queda en un medio estancado. Procesos de transporte más complejos, por ejemplo, análisis de la velocidad de caída en mezclas de sedimentos en suspensión requieren métodos más sofisticados como el uso de la técnica por Imágenes de Partículas (PIV) [19].

Los valores de las velocidades calculadas por la ecuación de Stokes sobrestiman de forma considerable los valores experimentales, debido que su aplicación se restringe a $R e_{p}<1$. Las velocidades calculadas por las ecuaciones de Julien y Rubey se mostrarón próximas a los valores experimentales, a pesar que las ecuaciones no consideran los factores de forma y redondez, de forma directa, pero fueron desarrolladas con sedimento de río lo que permitió ajustar el coeficiente de arrastre para este tipo de material.

Si se descarta el valor de la velocidad correspondiente a $d_{s}=1.6 \mathrm{~mm}$, la ecuación de Camenen muestra la segunda mejor predicción de las velocidades calculadas después de la ecuación de Julien. Por otro lado, los valores de velocidad ( $\operatorname{para} d_{s}=1.6 \mathrm{~m}$ ) realizados por [15] para temperaturas de $25^{\circ} \mathrm{C} \mathrm{y}$ $27^{\circ} \mathrm{C}$, son próximos a los valores calculados por las ecuaciones de Julien y Camenen. Por tal motivo, se considera que las ecuaciones de Julien y Camenen son las más indicadas para la determinación de la velocidad de caída del sedimento estudiado.

\section{AGRADECIMIENTOS}

A la Fundação de Amparo à Pesquisa de Minas Gerais (FAPEMIG) y a la Companhia Energética de Minas Gerais (CEMIG) por el financiamiento de la investigación.

\section{REFERENCIAS}

[1] G. Stokes. "On the effect of internal friction of fluids on the motion of pendulums". Trans. Cambridge Philos. Soc. Vol. 9, pp. 8-106. 1851.

[2] R.J. Hellermeir. "Fluvial Hydrodinamics: Sediment Transport and Scour Phenomena". Springer Heidelberg: New York, pp. 17-19. 1981. ISSN: 2190-5193.

[3] N.S. Cheng. "Simplified settling velocity formula for sediment particle". J. Hydraulic Eng. Vol. $123 \mathrm{~N}^{\circ} 2$, pp. 149-152. 1997. ISSN: 0733-9429.

[4] W. Rubey. "Settling velocities of gravel, sad and silt particles". Am. J. Sci. Vol. $25 \mathrm{~N}^{\circ}$ 148, pp. 325-338. 1933. ISSN: 0002-9599. DOI: 10.2475/ajs.s5-25.148.325.

[5] P.Y. Julien. "Erosion and Sedimentation". Cambridge: University Press, Segunda Edición, Cambridge, UK, pp. 94-98. 2010. ISBN: 978-0-521-53737-7.

[6] B. Camenen. "Simple and General Formula for The Settling Velocity of Particules". Journal of Hydraulic Engineering (C) ASCE, Vol. $133 \mathrm{~N}^{\circ}$ 2, pp. 229-233. 2007. ISSN: 0733-929. DOI: 10.1061.

[7] R. Soulsby. "Dynamics of marine sands: a manual for practical applications". Thomas Tel ford: London. 1997.

[8] J. Ahrens. "A fall-velocity equation". J. Waterway, Port, Coastal, Ocean Eng. Vol. $126 \mathrm{~N}^{\mathrm{o}}$ 2, pp. 99-102. March 2000. ISSN: 0733-950X.

[9] J. Guo. "Logarithmic matching and its applications in computational hydraulics and sediment transport". J. Hydraulic Res. Vol. $40 \mathrm{~N}^{\mathrm{o}}$ 5, pp. 555-565. 2002. DOI: $10.1080 / 00221680209499900$. 
[10] W. Dietrich. "Settling velocities of natural particles". Water Resour.Res. Vol. $18 \mathrm{~N}^{\circ} 6$, pp. 1615-1626. 1982. ISSN: 1944-7973.

[11] J. Jimenez and O. Madsen. "A simple formula to estimate settling velocity of natural sediments". J. Waterway, Port, Coastal, Ocean Eng. Vol. 129 N $^{\circ}$ 2, pp. 70-78. 2003. ISSN: 0733-950X.

[12] M. Zare and M. Koch. "Analysis of some common theoretical and empirical relationships between settlig velocity of a sediment particle as a function of particle size and water temperature and development of new empirical nolinear regression equations". 11th Conferência Internacional sobre Hydroscience \& Engineering, ICHE. Hamburgo, Alemania, 28 setembro - 2 outubro de 2014.

[13] J.Z. Tarqui. "Desenvolvimento de um método de fotografia para o estudo de fenômenos de altas frequências em aspersores jato-placa". Tesis para optar al grado de maestria. Universidade Federal de Uberlândia. Uberlândia, Brasil. 1996.

[14] J.L.Z. Tarqui, A. Silveira Neto and M. Harel. "Short Expousure Photographic Technique
Applied to Visualize a Free Liquid Jet". Journal of Flow Visualization Images Processing. Vol. 4 No 1, pp. 1-8. USA. 1997. DIO:10,1615/JFlowVisImageProc.v4.i1.

[15] A. Basile, G. Riccardi and I. Aguzzi. "Revisión de ecuaciones de velocidad de caída terminal de partículas de sedimento". Séptimo Simposio Regional sobre Hidráulica de Ríos. Cordoba, Argentina. 2015.

[16] W. Wu and S.S. Y. Wang. "Formulas for Sediment Porosity and Settling Velocity". Journal of Hydraulic Engineering. ASCE. Vol. 132 Issue 8, pp. 858-862.

[17] N.A. Riley. "Projection Sphericity". Journal of Sedimentary Petrology. Vol. 11 Issue 2, pp. 94-97. 1941.

[18] H. Wadell. "Volume, Shape and Roundness of Rock Particles". The Journal of Geology. Vol. 40, pp. 443-451. 1932.

[19] H. Salinas Tapia, J.A. García Aragón y C. Diaz Delgado. "Análisis experimental Del efecto de turbulencia en la velocidad de caída de sedimentos en suspensión". Ingeniería, Investigación y Tecnología. Vol. IX $\mathrm{N}^{\mathrm{o}} 1$, pp. 49-58. Enero-marzo, 2008. 